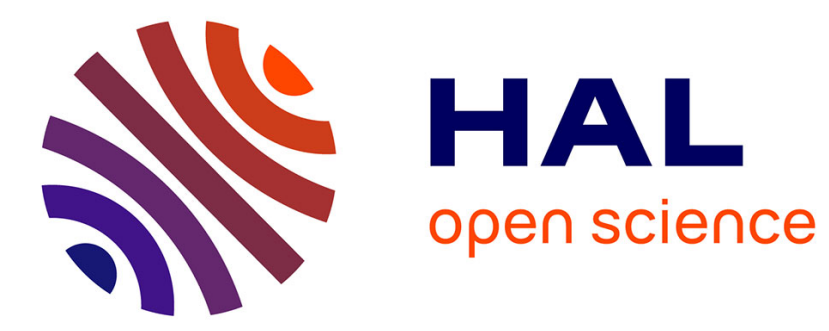

\title{
Modelling of aggregate restructuring in a weakly turbulent flow \\ Frédéric Gruy
}

\section{To cite this version:}

Frédéric Gruy. Modelling of aggregate restructuring in a weakly turbulent flow. Colloids and Surfaces A: Physicochemical and Engineering Aspects, 2012, 395, pp.54-62. 10.1016/j.colsurfa.2011.12.003 . hal-00667272

\section{HAL Id: hal-00667272 \\ https://hal.science/hal-00667272}

Submitted on 7 Feb 2012

HAL is a multi-disciplinary open access archive for the deposit and dissemination of scientific research documents, whether they are published or not. The documents may come from teaching and research institutions in France or abroad, or from public or private research centers.
L'archive ouverte pluridisciplinaire HAL, est destinée au dépôt et à la diffusion de documents scientifiques de niveau recherche, publiés ou non, émanant des établissements d'enseignement et de recherche français ou étrangers, des laboratoires publics ou privés. 


\title{
Modelling of Aggregate Restructuring in a Weakly Turbulent Flow
}

\author{
Frédéric GRUY \\ Ecole Nationale Supérieure des Mines, 158 Cours Fauriel, 42023 Saint-Etienne, \\ France, Cedex 2
}

Corresponding author :

Pr. Frédéric Gruy

Ecole Nationale Supérieure des Mines de Saint-Etienne

gruy@emse.fr

Tel 0033477420202

158 Cours Fauriel, 42023 Saint-Etienne, France, Cedex 2 


\begin{abstract}
An aggregate may undergo a restructuring that leads to a change of its porosity. The cause of this morphology change is not only the colloidal forces between the primary particles but also the interaction between the primary particles and the flowing fluid. We investigate in this paper the restructuring of the aggregates with Van der Waals forces between the primary particles in the presence of the Brownian motion and a weakly turbulent flow. The motion of all the primary particles constituting the aggregate is studied thanks to a 1-D model based on the work of G. Narsimhan [Model for drop coalescence in a locally isotropic turbulent flow field, J. of Colloid \& Interface Science, 272(2004)197-209]. A shrinking rate law, i.e. aggregate porosity versus time, is proposed after a transition to a 3-D model. Its characteristic time depends on the initial porosity, the number of primary particle and a characteristic time at the scale of the primary particle.
\end{abstract}

Keywords: aggregate, restructuring, shrinking time, turbulent flow field 


\section{Introduction}

Aggregation of small particles often occurs during industrial processes. The first step of this phenomenon is the collision of particles, which can be due to differential sedimentation, Brownian motion or shear flow in a duct or an agitated vessel. The size of the resulting clusters or aggregates increases with time. However, aggregates become looser and looser and undergo breakage. There is a competition between the cohesive forces inside the aggregate and the shear stress due to the liquid flow. Thus, the final size of aggregates can be calculated from a balance between aggregation and breakage. Aggregation and breakage have been investigated by many researchers [1]. Under certain operating conditions (supersaturation, mass transfer inside the aggregate ...), consolidation or strengthening of aggregates happens [2-7]. This takes place at the neck between adherent primary particles. The consolidation prevents the breakage of aggregates and leads to more porous clusters than the ones made without consolidation. At the same time, interaction between the flowing liquid and the aggregate can modify the internal structure of aggregates, breakage being the consequence of such a strong interaction. This results from the relative motion of primary particles inside the aggregates and leads to denser aggregates (see, for instance, [8-10]). Few works have been devoted to the study of the consolidation and the restructuring.

Several phenomena cause densification without consolidation: physical forces between primary particles, e.g. Van der Waals forces, Brownian motion of the primary particles leading to thermal restructuring, relative motion of the primary particles due to the shear flow, fragmentation-reaggregation steps, i.e. fragmentation of a large and loose aggregate followed by aggregation of the two fragments. At our knowledge, the modelling of these phenomena has not yet been performed. However, only attempts have been published. Investigators established aggregate restructuring laws from experiments and computer simulations.

\section{Empirical equations}

Several experimental works and computer simulations show that large aggregates have fractal morphology. In fact, an aggregate containing $i$ identical primary particles of radius $R$ is characterized by: the fractal dimension $D_{\mathrm{f}}$ and the outer diameter $R_{\mathrm{i}}$. These two parameters are linked by the following relations:

$$
R_{i}=R(i / S)^{1 / D_{f}}
$$

The structure factor $S$ is depending on $D_{f}$. Its value is close to 1 .

As a first approximation, clusters keep the same fractal dimension along the aggregation process. However, it follows from some experimental evidence [8-10] that the fractal 
dimension value increases with time. So the simplest way for describing this observation makes use of a relaxation law:

$d D_{f} / d t=\left(D_{\max }-D_{f}\right) / \tau$

$D_{f}, D_{\max }, \tau$ are respectively the actual fractal dimension, its maximum value and a relaxation time. The relaxation time includes the above-mentioned physical contributions.

Relaxation time may have a constant value [11], may be a function of the actual aggregate size [12], or may be a function of the mean aggregate size at a given time [13-15]. The relaxation time [12-15] is expressed by means of empirical equations containing fitted parameters. So, 2 or 3 fitted parameters are used for each physical contribution.

Selomulya et al. [13-14] propose:

$$
\tau^{-1}=c_{1} \bar{i}^{n}+c_{2}\left(\sum_{i}\left|d n_{i} / d t\right|_{A}\right)\left(\sum_{i}\left|d n_{i} / d t\right|_{F}\right)
$$

$\mathrm{c}_{1}, \mathrm{c}_{2}$ are constants. $n_{i}$ represents the number concentration in i-mer. $\mathrm{A}$ and $\mathrm{F}$ are relative to aggregation and fragmentation processes.

Baldyga et al. [15] propose:

$$
d D_{f} / d t=C_{\gamma} \dot{\gamma}\left(D_{\gamma, \max }-D_{f}\right)+C_{A} K_{A} M_{0}\left(D_{A, \min }-D_{f}\right)+C_{F} K_{F}\left(D_{F, \min }-D_{f}\right)
$$

$C_{\gamma}, C_{A}, C_{F}$ are constants. $\mathrm{K}_{\mathrm{A}}$ and $\mathrm{K}_{\mathrm{F}}$ are agglomeration and fragmentation kernels. $M_{0}$ is the zero-order moment of the particle size distribution. $\dot{\gamma}$ is the shear rate. $D_{A \text {,min }}, D_{F, \text { min }}$ are fitted fractal dimensions.

Kostoglou et al. [12] suggest:

$\tau \propto i^{p}$

The value of the asymptotic fractal dimension is also fitted:

$D_{\max }>2.5$

These empirical equations well represent the reality, but can be only applied to the considered systems.

\section{Simulations}

Starting from assumed restructuring mechanisms at the primary particle scale, and being given an initial aggregate morphology, computer simulations lead to the morphology of the aggregate at a given time. As a result, the relaxation time can be deduced. So, in the case of thermal restructuring, Dalis [16] shows that the relaxation time depends on the temperature, the size and the number of primary particles in the aggregate: 
$\tau \propto f(i, R, T)$

The computer simulations also give the maximum value of fractal dimension.

Simulation of aggregation has been extensively studied [1]. We mention the work of Gmachowski [17] as he proposes a new approach of the aggregation modelling by considering an aggregation act for which the fractal dimension can vary along the process. The master equation is the following:

$(i+j)^{1 / D}=F\left(i^{1 / D_{i}}+j^{1 / D_{j}}\right)$

$D_{i}, D_{j}, D$ are the fractal dimension of $\mathrm{i}$-mer, $\mathrm{j}$-mer, $(\mathrm{i}+\mathrm{j})$-mer. $F$ can be expressed as a function of the space dimension, the trajectory dimension and $D$. Restructuring may be taken into account [18] by changing the value of $F$. Kostoglou and Konstandopoulos [19] use a similar aggregation act for studying Brownian aggregation.

Higashitani et al. [20] present a three dimensional discrete element method (DEM) to simulate the deformation and break-up of large aggregates in flowing viscous fluid (dynamic viscosity $\mu$ ). They take into account the Van der Waals forces between submicronic primary particles, the drag forces acting on the particle surface exposed directly to the flow, and the elastic interaction between primary particles inside the aggregate. The model is applied to the behaviour of the aggregates in shear and elongational flows for $\mu \dot{\gamma}>50 \mathrm{~Pa}$. Shear flow results in rotation, deformation and break-up of the aggregates. The averaged number of primary particles in the resulting fragments is only dependent on the ratio of the hydrodynamic drag force and the adhesive force at the scale of a two primary particle set:

$$
\langle i\rangle=27.9\left(\frac{6 \pi \mu R^{2} \gamma}{A R / 12 \Delta^{2}}\right)^{-0.872}
$$

where $\Delta$ is the minimum separation distance between primary particle surfaces and $A$ is the Hamaker constant.

Zeidan et al. [21] consider the deformation and the fragmentation of $2 \mathrm{D}$ aggregates composed of sub-millimetric particles in a shear flow. Simulations are carried out by a combined approach of discrete element method and computational fluid dynamics. Van der Waals forces and drag forces are also considered. As expected, the ratio between the shear stress and the adhesive stress drives the dynamics of the morphology changes: for high ratio values, elongation and break-up dominate, whereas rotation, rounding and erosion occur for small ratio values. 
Tatek et Pefferkorn [22] studied the cluster-cluster aggregation taking into account the connectivity inside the aggregate. Aggregation is described by all classical ways except the use of a stability criterion:

if $\frac{i}{j}(i+j) \frac{1}{v}<K$ then the collision (with $i<j$ ) is efficient.

$v$ is the contact number between the two colliding aggregates. The $K$ constant is chosen by the user. This criterion is closely related to an aggregation-fragmentation mechanism for the restructuring. They observe that the fractal dimension is a decreasing function of $K$.

\section{Population balance equation and restructuring}

Population balance equation (PBE) is a partial differential equation that gives the population density. The latter is a function of the time $t$ and internal parameters for homogeneous suspension. The phenomena acting on the population density are the nucleation, growth, agglomeration and breakage. The corresponding terms are classically included in the PBE. To take into account the aggregate restructuring a convective term including the restructuring law can be introduced in the PBE. So, 1-D PBE (one internal parameter) or 2-D PBE (two internal parameters) has been proposed by investigators [11-15]. Thus, the restructuring law, i.e. aggregate porosity or fractal dimension versus time, plays a crucial role in the modelling of the aggregation dynamics.

The purpose of this paper is to establish the restructuring laws for aggregates formed in the conditions of perikinetic and orthokinetic aggregations. The section two presents the framework of the modelling of the restructuring and preliminary results. Emphasis is given to the restructuring in a motionless fluid. The section three is devoted to the search of restructuring laws for aggregates in a weakly turbulent flow. As a conclusion the results will be discussed in the section four. 


\section{Restructuring by attractive interaction between primary particles}

\subsection{Modelling}

Let us consider the restructuring of an aggregate by attractive interaction between primary particles. Each primary particle undergoes attractive forces due to the other particles and the drag force in a still fluid. The restructuring will be considered as isotropic. As a consequence, we will study the behaviour of a particle chain along a radial line of the aggregate. We consider here that the orthoradial interactions have a negligible effect on the motion of primary particles. This point will be discussed in $\S 2.3$ and 3.4. The figure 1 shows the chain of $N$ mono-sized primary particles. The particles are assumed as spherical with the radius $R$. The primary particle at the center of the aggregate (denoted 1) will be located at one (left) end of the chain. It will be motionless by symmetry. The location of a given particle $\mathrm{i}$ is denoted by $x_{i}$ that is the distance between the centers of the actual particle and the particle 1 . The primary particle at the (right) end of the chain is located at the surface of the aggregate.

A given primary particle $i$ undergoes the physicochemical interaction with its nearest neighbours $i-1$ and $i+1$. The attractive interaction is due to Van der Waals forces. The interaction potential between two primary particles separated by the center-to-center distance $r$ is expressed thanks to Hamaker [1]:

$$
V(r)=-\frac{A}{6}\left[\frac{2 R^{2}}{r^{2}-4 R^{2}}+\frac{2 R^{2}}{r^{2}}+\ln \frac{r^{2}-4 R^{2}}{r^{2}}\right]
$$

$A$ is the Hamaker constant of the system constituted by the particle material and the fluid.

The Van der Waals force obeys:

$$
F=-d V(r) / d r
$$

The Newton's second law is applied to the particle $\mathrm{i}$ :

$m \frac{d^{2} x_{i}}{d t^{2}}=F\left(h_{i-1}\right)-F\left(h_{i}\right)-6 \pi \mu R \frac{d x_{i}}{d t}$

with

$h_{i}=x_{i+1}-x_{i}$

As the particle Reynolds number is much smaller than 1, the drag force obeys the Stokes law. $\mu$ is the dynamic viscosity of the fluid.

By rendering the Eq.9a-b dimensionless: 
$H \frac{d^{2} x_{i}^{\prime}}{d t^{\prime 2}}=F^{\prime}\left(h_{i-1}^{\prime}\right)-F^{\prime}\left(h_{i}^{\prime}\right)-\frac{d x_{i}^{\prime}}{d t^{\prime}}$

with

$t=t^{\prime} \tau \quad \tau=72 \pi \mu R^{3} / A$

$H=\frac{\rho_{s} A}{324 \pi \mu^{2} R}$

$F^{\prime}\left(r^{\prime}\right)=\frac{4 r^{\prime}}{r^{\prime 2}-4}-\frac{8 r^{\prime}}{\left(r^{\prime 2}-4\right)^{2}}-\frac{8}{r^{\prime 3}}-\frac{4}{r^{\prime}}$

The dimensionless distances and the time are obtained respectively by the radius of the primary particle and $\tau$. Usually $H$ is much smaller than 1 . As a consequence we will consider inertialess particles:

$\frac{d x_{i}^{\prime}}{d t^{\prime}}=F^{\prime}\left(h_{i-1}^{\prime}\right)-F^{\prime}\left(h_{i}^{\prime}\right) \quad i>1$

Along the time course the primary particles collide inside the aggregate and form clusters as the particles become bound after the collision. The cluster size, i.e. the number of primary particles in the cluster, is increasing and the number of clusters is decreasing. Thus the interaction between clusters and not only the interaction between single primary particles will be considered (Figure 2).

The force between two clusters denoted $i$ and $i+1$ is the sum of all inter-particle forces:

$F_{i, i+1}^{a}=\sum_{m_{i}=0, m_{i+1}=0}^{M_{i}-1, M_{i+1}-1} F^{\prime}\left(h_{i}^{\prime}+2 m_{i+1}+2 M_{i}-2-2 m_{i}\right)$

$M_{i}$ is the number of primary particles inside the i cluster. $h_{i}^{\prime}$ is the distance between the centre of the last (right) particle of the i cluster and the center of the first (left) particle of $i+1$ cluster. A given cluster is marked by the coordinate of the center of its first (left) primary particle.

The dimensionless drag for a cluster may be calculated following Batchelor [23] and Rogak and Flagan [24]:

$T_{i}^{\prime}=-\Omega_{i} \frac{d x_{i}^{\prime}}{d t^{\prime}}$

with

$\Omega_{i}=\frac{2}{3}\left(M_{i}^{2}-1\right)^{1 / 2}\left[\frac{\varepsilon+0.307 \varepsilon^{2}}{1-0.5 \varepsilon}+0.426 \varepsilon^{3}\right]$

and

$\varepsilon=1 / \operatorname{Ln}\left(2 M_{i}\right)$ 
However the Stokes law is valid only for a cluster that is kept away from its neighbours. The lubrication theory leads to a corrective term for the Stokes law that takes into account the additional hydrodynamic resistance due to the proximity of the other particles.

We will use the approach of Zeichner and Schowalter [25]: the attractive force on the particle i due to the particle $i+1$ is reduced by a factor:

$G_{i, i+1}=\left(h_{i}^{\prime}-2\right) /\left(h_{i}^{\prime}-2+C\right)$

where $C$ is a constant whose value is equal to 0.25 .

Finally the motion of the cluster i obeys the expression:

$\frac{d x_{i}^{\prime}}{d t^{\prime}}=\left(F_{i-1, i}^{a}\left(h_{i-1}^{\prime}\right) G_{i-1, i}-F_{i, i+1}^{a}\left(h_{i}^{\prime}\right) G_{i, i+1}\right) / \Omega_{i} \quad i>1$

with

$x_{i}^{\prime}=\sum_{j=1}^{j=i-1} 2 M_{j}-2+h^{\prime}{ }_{j} \quad i>1$

A recursive computational program was involved to solve Eqs.16a-b. Suppose $N_{a}$ be the cluster number in the aggregate at a given time; Eqs 16a-b are solved till two clusters collide. As a consequence a new configuration of the chain appears with $N_{a^{-}} 1$ clusters. This procedure is repeated until a single cluster is obtained.

\subsection{Results}

Generally 3D-aggregates contain a finite number $\left(<10^{5}\right)$ of primary particles. Thus, the number $N$ of primary particles inside the corresponding 1D-chain will be chosen smaller than 40. We examine the case of initially identical inter-particle distances.

The figure 3 shows the change with time of the morphology of a chain with 30 primary particles. A set (line) of horizontal dots represents the chain at a given time. Each line corresponds to a given dimensionless time. At time zero the dimensionless inter-particle distance is equal to 3 , i.e. the surface-surface distance is equal to the particle radius.

The restructuring of the aggregate happens by successive steps that can be a new location of primary particles either without global shrinking or with shrinking.

Simulations have been performed with:

- $\quad N:[4,6,10,20,30,40]$

- $\quad h^{\prime}(t=0):[3,4,6]$ i.e. $\phi_{0}=2 / h_{0}^{\prime}:[0.667,0.5,0.333]$

Time and position of each primary particle in a given chain are recorded at each collision. These records are achieved for each set of $N, h^{\prime}{ }_{0}$. The data are transformed into a graph 
representing the mean solid volume fraction $\phi$ in the aggregate as a function of the time. The figure 4 shows the corresponding curves for $h^{\prime}{ }_{0}=1$ and various $N$.

Finally all the simulation results may be drawn on a unique graph (figure 5).

$X$ and $Y$ are defined as:

$X=t^{\prime}\left[L_{0}\left(1-\phi_{0}\right)\right]^{-5.5}$

$Y=\left(\phi-\phi_{0}\right) /\left(1-\phi_{0}\right)$

$L_{0}$ is the aggregate size, i.e. the centre to centre distance between the first and the last primary particles at time zero. $Y$ is within the [0-1] range along the restructuring process. $L_{0}\left(1-\phi_{0}\right)$ is the porous volume (normalized by the radius of the primary particle) of the aggregate: several parameters have been tested and this one was the more relevant and efficient parameter for linking the various quantities of the system. Moreover it is expected that the porous volume is an important parameter for the restructuring dynamics.

The non-unique interpolation function can be expressed:

$Y=1-e^{-3 X^{0.4}}$

The figure 5 shows that the shrinking of the aggregate is negligible when $X<10^{-2}$, but will be completed at $\mathrm{X} \approx 0.1$, i.e. for the dimensionless time:

$t_{F}{ }^{\prime} \approx 0.1\left[L_{0}\left(1-\phi_{0}\right)\right]^{5.5}$

As a conclusion, one observes two successive stages: a restructuring without shrinking and the shrinking itself.

\subsection{Effect of the Brownian motion}

If the aggregate is composed of nanoparticles it may be expected that the Brownian motion significantly contributes to the restructuring.

The motion of a nanoparticle in a fluid obeys the Langevin equation:

$m d v(t)=-f v(t) d t+d W_{t}$

$v$ is the velocity of the particle. $W_{t}$ is a Wiener process with a mean value zero and a standard deviation obeying $f^{2} 2 D d t$.

$f$ is the drag coefficient (following Stokes: $f=6 \pi \mu R$ ). $D$ is the diffusion constant ( $D=k T / f$ ). The Langevin equation means that a driving force $d W_{t} / d t$ due to random collisions between the particle and the fluid molecules is counterbalanced by a friction force $-f v(t)$. 
Then,

$m d v(t)=-f v(t) d t+f \sqrt{2 D d t} R_{n}$

$R_{n}$ is a function that generates a random number for a Gaussian distribution with a mean value zero and a standard deviation equal to 1 . We introduce the shortened notation $\sqrt{d t}$ for practical reason as the standard deviation of the stochastic process is proportional to $d t$.

If the inertia of the particle is negligible, then:

$0=-f d x+f \sqrt{2 D d t} R_{n}$

Let us consider a motionless particle and a Brownian particle interacting by a colloidal force $F$. The centre to centre distance $h(t)$ obeys the equation ([26]):

$d h(t)=\left(\frac{F(h)}{f}\right) d t+d W_{t}$

with

$d W_{t}=\sqrt{2 D d t} R_{n}$

By taking into account the hydrodynamic resistance between two primary particles (Eq.15) and after dimensionless transformation:

$d h^{\prime}=\left(F^{\prime}\left(h^{\prime}\right) d t^{\prime}+b\left(d t^{\prime}\right)^{1 / 2} R_{n}\right) G\left(h^{\prime}\right)$

$b=(24 k T / A)^{1 / 2}$

The $b$ value is equal to about $3\left(A \approx 10^{-20} J ; T=300 K\right)$.

From Eq.23a and Eq.16a one may deduce the stochastic equation for the motion of the cluster $\mathrm{i}$ taking into account the Van der Waals forces and the Brownian motion:

$d x_{i}^{\prime}=\left(F_{i-1, i}^{a}\left(h^{\prime}{ }_{i-1}\right) G_{i-1, i}-F_{i, i+1}^{a}\left(h_{i}^{\prime}\right) G_{i, i+1}\right) / \Omega_{i} d t^{\prime}+b \Omega_{i}^{-3 / 2} G_{j, j+1}\left(d t^{\prime}\right)^{1 / 2} R_{n} \quad 1<i \leq N_{a}$

$N_{a}$ is the index of the farthest (last) cluster (or primary particle) from the center of the aggregate. $G$ is the hydrodynamical resistance that is depending on the inter-particle distance. The value of the index $j$ is discussed below.

The Brownian motion moves the particle or cluster in two directions: the particles may go away or come near again. Practically a maximum value for the size $x_{N}$ of the aggregate has to be imposed along the simulations: if the size exceeds this value the simulation stops. Larger the maximum size value is, larger is the number of simulations leading to the total shrinking of the aggregate. One thousand simulations were performed for each chain configuration. 
They were compared one to one to the deterministic case $(b=0)$. The distribution of the restructuring time has been deduced.

Two kinds of simulations corresponding to different conditions have been tested:

a. The last particle or cluster $i=N_{a}$ undergoes Brownian motion as the core particles or clusters do. As a consequence a great expansion of the aggregate may happen (the aggregate size can be multiplied by ten). The maximum size value will be fixed as:

$x_{N}^{\operatorname{Max}}=K x_{N}(t=0)$

where $K$ is a constant.

then for $i \leq N_{a}$

$$
\begin{array}{lll}
j=i-1 & \text { if } & R_{n}<0 \\
j=i & \text { if } & R_{n}>0
\end{array}
$$

The table 1 reports the ratio $P$ of simulations leading to the total shrinking and the median value $T$ of the shrinking time for several $K$ values. The studied aggregate contains four primary particles. It can be observed a quasi-proportionality between $K$ and $T$. These $T$ values have to be compared to the deterministic case $(T=147)$.

b. The last primary particle or cluster does not undergo Brownian motion. This procedure maintains the wholeness of the aggregate; the aggregate size is always decreasing $(\Rightarrow P=1)$. This strict condition is coming from the lateral and strong bonds between the primary particles at the surface of the aggregate, and introduces a purely tridimensional hindrance. This is the condition for the existence of the aggregate. Thus we assume:

$$
\begin{array}{lll}
\text { for } i<N_{a} & & \\
j=i-1 & \text { if } & R_{n}<0 \\
j=i & \text { if } & R_{n}>0
\end{array}
$$

and $G_{N a-1, N a}=0$

The figures 6a-b show the distribution density of the total shrinking time (for $\mathrm{N}=4$ ) and the distribution density of the shrinking time at an intermediate event when the aggregate consists of two clusters. The median value of the shrinking time is equal to 167.9 (the mean value is equal to 184 ) for a 4-aggregate.

The two cases lead to two different results: however the second one (b) seems more consistent with a 3-D restructuring. Whatever the porosity and the number of primary particles the 
Brownian motion increases the shrinking time by a factor equal to 1.5-3 whereas the intermediate shrinking time is decreased.

The removal of the constraint, i.e. the finite K-value, may represent the break-up of the aggregate due to Brownian motion. However it is assumed along the paper that the dominant phenomenon is the attractive interaction. As a consequence the break-up would be negligible. It can be argued that the break-up will occur when the value of a selected parameter reaches a critical value. This parameter could be the $\mathrm{K}$ parameter. However a dimensionless parameter like the ratio between $k T$ and $\min \left\{\left|V_{i, i+1}^{a}\left(h_{i}^{\prime}\right)\right|\right\}$ (interaction potential between $\mathrm{i}$ and $\mathrm{i}+1$ clusters) would be more suitable.

\section{Restructuring in a turbulent flow}

\subsection{Modelling}

Narsimhan [26] has studied the coalescence of two droplets which are close to each other in a turbulent flow. The two droplets undergo colloidal forces and a turbulent force $F_{T}$. The latter one is attractive and fluctuating. Its expression depends on the turbulence range:

$$
F_{T}=\overline{F_{T}}-\delta T^{1 / 2} S(t)
$$

With the standard deviation $\delta$ such as $\delta=\overline{F_{T}}$ and

$$
S(t) d t=d W_{t} .
$$

$W_{t}$ is a Wiener process with a mean zero and a standard deviation $d t$. In the viscous range of the turbulence, $T$ and $\overline{F_{T}}$ obey:

$$
T=(v / \varepsilon)^{1 / 2}
$$

and

$\overline{F_{T}}=\pi R^{2} \rho(\varepsilon / v)(h+2 R)^{2}$

$\varepsilon$ is the turbulence dissipation rate.

The approach of Narsimhan is applied to the restructuring of an aggregate in a weakly turbulent flow. As the aggregate size is much smaller than the Kolmogorov scale, the restructuring takes place in the viscous range of the turbulence.

The relative velocity of two inertialess particles is proportional to the sum of the forces: the colloidal force $F$, the Brownian force and the turbulent force. Then, Eq.23a becomes:

$$
d h^{\prime}=\left(F^{\prime}\left(h^{\prime}\right) d t^{\prime}-b_{1}\left(2+h^{\prime}\right)^{2} d t^{\prime}+\left(b_{2}\left(2+h^{\prime}\right)^{2} R_{n, 2}+b_{3} R_{n, 3}\right)\left(d t^{\prime}\right)^{1 / 2}\right) G\left(h^{\prime}\right)
$$


The two stochastic (Brownian and turbulent) processes are assumed to be independent. $R_{n}$ is a function that generates a random number for a Gaussian distribution with a mean value zero and a standard deviation equal to 1 .

The $b_{1}, b_{2}$ et $b_{3}$ dimensionless parameters are expressed as:

$b_{1}=12 \pi R^{5} \rho(\varepsilon / v) / A$

$b_{2}=\rho(\varepsilon / v)^{3 / 4}\left(2 \pi R^{7} /(\mu A)\right)^{1 / 2}$

$b_{3}=b$

The previous modelling $(\S 2.1, \S 2.2)$ corresponds to $b_{1}=b_{2}=b_{3}=0$.

The extension of the modelling of the two-particle set motion (Eq.26) to the one of the particle chain follows the methodology leading to Eq.24 for Brownian motion:

$$
\begin{aligned}
& d x_{i}^{\prime}=\left(\left(F_{i-1, i}^{a}\left(h_{i-1}^{\prime}\right)-b_{1}\left(2+h_{i-1}^{\prime}\right)^{2}\right) G_{i-1, i}-\left(F_{i, i+1}^{a}\left(h_{i}^{\prime}\right)-b_{1}\left(2+h_{i}^{\prime}\right)^{2}\right) G_{i, i+1}\right) \Omega_{i}^{-1} d t^{\prime} \\
& +b_{2}\left(\left(2+h_{i}^{\prime}\right)^{2} A_{i} R_{n, 2}^{(i)}-\left(2+h_{i-1}^{\prime}\right)^{2} A_{i-1} R_{n, 2}^{(i-1)}\right) \Omega_{i}^{-1}\left(d t^{\prime}\right)^{1 / 2}+b_{3} \Omega_{i}^{-3 / 2} G_{j, j+1} R_{n, 3}\left(d t^{\prime}\right)^{1 / 2}
\end{aligned}
$$

With $A_{k}=G_{k, k+1}$ if $R_{n, 2}^{(k)}>0$ and $\quad A_{k}=1$ if $R_{n, 2}^{(k)}<0$

We also assume that:

- all the particles or the clusters of the chain undergo Brownian motion except the last one

- only the last cluster but one $N_{a}-1$ and the last cluster $N_{a}$ undergo the turbulence The reasons are the following:

- The Brownian motion is due to the collision between fluid molecules and particles. All the particles of the aggregate undergo this phenomenon. However, as mentioned in $\S 2.3$, the non-Brownian motion of the last particle guarantees the wholeness of the aggregate.

- The main effect of the turbulence is expected on the particles close to the surface: the internal particles are shielded by the external particles. As shown below, taking into account the effect of the turbulence on the internal particles do not significantly modify the results of the simulations.

\subsection{Results}

Simulations have been realized for chains consisting of initially equidistant particles. The physical parameters are $R, \mathcal{\varepsilon}, N$ and $h_{0}$. Their value ranges are: 
$0.5 \mu m<R<5 \mu m$

$0.01 m^{2} s^{-3}<\varepsilon<10 m^{2} s^{-3}$

$0.5 R<h_{0}<4 R$

$4<N<40$

The figure 7 shows the change with time of a chain having 30 primary particles. It can be observed that the shrinking of the aggregate is a progressive process unlike the previous case (fig. 3).

Among the three dimensionless parameters, only $b_{1}$ is a relevant parameter: the ratio $b_{2} / b_{1}$ is always smaller when the turbulence is taken into account, and $b_{3}$ has a small effect on the restructuring in a turbulent flow.

We define the new dimensionless variables:

$$
\begin{aligned}
& X=t^{\prime}\left[L_{0}\left(1-\phi_{0}\right)\right]^{-3 / 2} \\
& Y=\left(\phi-\phi_{0}\right) /\left(1-\phi_{0}\right) \\
& B=\phi_{0}^{-3 / 2} b_{1}^{2 / 3}
\end{aligned}
$$

The figure $8 \mathrm{a}$ shows the variable $Y$ as a function of $B(1+0.05 B) X$. Each dot represents a simulation performed for a given set of $R, \varepsilon, N$ and $h_{0}$ parameters. The dots set is spread, but no dependence on the $B$-range can be observed. So, by using these new variables we have:

$$
Y=4.5 B(1+0.05 B) X \quad \text { if } \mathrm{B}<25
$$

Or as a first approximation:

$$
Y=6.5 B X
$$

The Eqs. 29c, 30a-b are obtained by an optimization procedure.

Simulations have been also realized for initially fractal aggregates with the fractal dimension values: $\mathrm{D}_{\mathrm{f}}=1.8$ and $\mathrm{D}_{\mathrm{f}}=2$.2. A linear relation between $Y$ and $B(1+0.05 B) X$ is also verified. However the proportionality coefficient is slightly different and equal to 5.6:

$$
Y=5.6 B(1+0.05 B) X
$$

From Eq.30a and Eq.31, we will retain the following expression whatever the aggregate morphology:

$Y=5 B(1+0.05 B) X$

A simulation has been realized as all the primary particles undergo the fluctuating shear whereas the previous simulation (figure 8a) has been realized as only the external primary particles undergo the fluctuating shear. The comparison of the two simulations confirms that 
the effect of the turbulence on the internal primary particles has no consequence on the shrinking process. It can be simply noted that there is a slight discriminating effect of the $B$ range on the function $Y=f(B(1+0.05 B) X)$.

\subsection{Approximated expression}

We will perform some simplifications on the Eq. 26 that are justified by a thorough study:

- Cancellation of the stochastic terms

- Approximating $F^{\prime}$ by $F^{\prime}=-h^{1^{-2}}$ : this approximation is only valid for small values of h' whereas the magnitude of the colloidal force is much smaller than the turbulent force for high values of h'.

- Performing successive applications of Eq. 33 to the last inter-particle spacing (denoted $\left.j=N_{a}-1\right)$ as the primary particle $j$ is motionless whereas the cluster $N_{a}$ with $N-j$ primary particles is mobile with a drag coefficient $\Omega_{j+1}$ :

$$
d h^{\prime}=\left(-h^{\prime-2}-b_{1}\left(2+h^{\prime}\right)^{2}\right) h^{\prime} /\left(0.25+h^{\prime}\right) / \Omega_{j+1} d t^{\prime}
$$

The first shrinking step corresponds to $j=N-1$.

Thus the shrinking duration (for the $j$-spacing) is:

$$
\tau_{j}=\int_{h_{0 j}^{\prime}}^{0}\left[\left(-h^{\prime^{-2}}-b_{1}\left(2+h^{\prime}\right)^{2}\right) h^{\prime} /\left(0.25+h^{\prime}\right) / \Omega_{j+1}\right]^{-1} d h^{\prime}
$$

As the shrinking consists of the series of events with the shrinking duration:

$$
\begin{aligned}
T_{k} & =\sum_{j=N-k}^{N-1} \tau_{j}, x_{k}=2 k+\sum_{i=1}^{N-k-1}\left(2+h_{i, 0}\right) \quad 1 \leq k \leq N-2 \\
T_{N-1} & =\sum_{j=1}^{N-1} \tau_{j}, x_{N-1}=2(N-1)
\end{aligned}
$$

The total duration of the aggregate shrinking is $T=T_{N-1}$.

$h_{0, j}^{\prime}$ is the initial distance between the primary particles $j$ and $j+1$. If this one has the same value $h_{0}^{\prime}$ (whatever $j$ ), then:

$$
T=\int_{h_{0}^{\prime}}^{0}\left[\left(-h^{\prime^{\prime}-}-b_{1}\left(2+h^{\prime}\right)^{2}\right) h^{\prime} /\left(0.25+h^{\prime}\right)\right]^{-1} d h^{\prime} \sum_{k=1}^{N-1} \Omega(k)
$$

$k$ is the number of primary particles in the cluster. $\Omega(k)$ is given by Eqs. $14 \mathrm{~b}-\mathrm{c}$ with $M_{i}=k$. 
The figure $8 \mathrm{~b}$ represents the variable $Y$ as a function of $B(1+0.05 B) X$ by applying Eqs 3435. The same ranges of the various parameters have been used for the two calculations (Fig.8a and $8 \mathrm{~b}$ ). The figures $8 \mathrm{a}$ and $8 \mathrm{~b}$ are very similar. The approximated expression Eq.36 is in agreement within $20 \%$ with Eq. 32 . The scattering of the dots on the figures $8 a-b$ is due at once to the stochasticity of the phenomena and to the simplicity of the modelling (Eq.32). The corresponding standard deviation is about $40 \%$.

\subsection{From 1D model to 3D model}

Equation 32 gives the change of the aggregate radius with time. Solid volume fractions $\phi_{0}, \phi$ are expressed in a 1-D space. The relationship between the 1-D volume fraction $\phi$ and the 3D volume fraction $\Phi$ is:

$\Phi=\phi^{3}$

The equations 29a-c and 32 become:

$B=\Phi_{0}^{-1 / 2} b_{1}^{2 / 3}$

$\left(\Phi^{1 / 3}-\Phi_{0}^{1 / 3}\right) /\left(1-\Phi_{0}^{1 / 3}\right)=t^{\prime} / t_{F}{ }^{\prime}=t / t_{F}$

with

$t_{F}{ }^{\prime}=[5 B(1+0.05 B)]^{-1} V_{0}^{1 / 2}(2 R)^{-3 / 2}\left(1-\Phi_{0}^{1 / 3}\right)^{3 / 2}$

Then the total shrinking time obeys:

$t_{F} \approx 4 \mu /\left(A R \rho^{2}(\varepsilon / v)^{2}\right)^{1 / 3} N^{1 / 2}\left(1-\Phi_{0}^{1 / 3}\right)^{3 / 2}$

The most important parameters are practically the shear rate $\dot{\gamma}=(\varepsilon / v)^{1 / 2}$ and the number $N$ of primary particles. For typical parameter values ( $A=10^{-20} \mathrm{~J} ; R=1 \mu \mathrm{m} ; \rho=10^{3} \mathrm{Kgm}^{-3} ; \mu=10^{-3} \mathrm{~Pa} . \mathrm{s} ; \varepsilon=0.1 \mathrm{~m}^{2} \mathrm{~s}^{-3}$ ), the total shrinking time is equal to $t_{F}=335 \mathrm{~s}$ for $\Phi_{0}=0.1 ; N=10^{4}$ and $t_{F}=25 \mathrm{~s}$ for $\Phi_{0}=0.5 ; N=10^{3}$. These values are multiplied by 4.6 for smaller turbulent dissipation rate $\varepsilon=0.01 \mathrm{~m}^{2} \mathrm{~s}^{-3}$.

The restructuring time has to be compared to the aggregation time which obeys $t_{A}=5 /(\dot{\gamma} \Psi)$ [27]. $\Psi$ is the solid volume fraction in the aggregating suspension. Typical value for $\Psi$ in a aggregation experiment is $\Psi=10^{-4}$. Thus the aggregation time is about 500s for $\mathcal{E}=0.01 \mathrm{~m}^{2} \mathrm{~s}^{-3}$ whereas the restructuring time is about 500s for large aggregate $\left(N=10^{4}\right)$ and 50s for small aggregate $\left(N=10^{2}\right)$. Starting from a mono-disperse suspension and for these 
representative parameters, the restructuring simultaneously occurs with the aggregation. Depending on the parameters values the restructuring rate can be faster or slower than the aggregation rate. Accordingly the aggregates will be more compact or more porous.

The proposed 1D-3D extension is a bound for real systems. The main assumption in the one dimensional model is the autonomous behavior of a particle chain located along the aggregate radius. From symmetry arguments this represents well the effect of physical forces in a three dimensional aggregates. However we don't take into account the steric hindrance due to the (orthoradial) neighboring particles. This effect is an increasing function of the internal solid volume fraction that has a high value at the end of the restructuring process. Thus the model overestimates the restructuring rate. During the competition between restructuring and aggregation in a suspension, lower the aggregation rate (for instance for low particle concentration) more completed is the restructuring; as a consequence our model does not well represent the suspension dynamics in this case. In contrast, if the aggregation is rapid our model of restructuring is valid.

\section{Discussion and conclusion}

The simulations of the restructuring of an aggregate show that the restructuring of the aggregate by only attractive Van der Waals forces begins by the rearrangement of particle set. This rearrangement goes on until the formation of two clusters, and is followed by the shrinking of this two-cluster set. Stochastic phenomena drastically change the sequence of restructuring. Brownian motion slows the shrinking or even destroys the aggregate. The restructuring of an aggregate in a weakly turbulent flow is a progressive shrinking that depends on morphological parameters as $N$ and $\Phi_{0}$ but also on a characteristic time $\tau_{1}=\mu /\left(A R \rho^{2}(\varepsilon / v)^{2}\right)^{1 / 3}$ at the scale of the primary particle. Eqs.37b and 38 allow to calculate the porosity of the aggregate as a function of time. It is interesting to look for a physical meaning for $\tau_{1}$. One may use the classic dimensionless numbers in aggregation theory [1]: $C_{A}=A /\left(36 \pi \mu \dot{\gamma} R^{3}\right)$ and $\operatorname{Re}_{p}=\rho(2 R)^{2} \dot{\gamma} / \mu \quad$ (with $\dot{\gamma}=(\varepsilon / v)^{1 / 2}$ ). Then, $\tau_{1}=(9 \pi / 4)^{-1 / 3} C_{A}^{-1 / 3} \operatorname{Re}_{p}^{-2 / 3} \dot{\gamma}^{-1}$. The shear rate inverse is the time scale, $\operatorname{Re}_{p}$ is the Reynolds number based on the relative velocity between two colliding primary particles and $C_{A}$ is directly connected to the aggregation efficiency. 
Comparison of the present modelling with experimental results coming from the literature only concerns a few published studies. The latter ones propose expressions or values for the relaxation time $\tau$ present in Eq.2. Baldyga et al. [15] suggested for silica aggregates in a high shear device $\tau=10^{5} \dot{\gamma}^{-1}$ with a rough accuracy. Selomulya et al. [14] found out $\tau=50(N / S)^{-0.1 / D_{f}}$ for aggregates consisting of small polystyrene beads $\left(\mathrm{R}=0.405 \mu \mathrm{m} ; \dot{\gamma}=100 s^{-1}\right)$ and $\tau=200 \dot{\gamma}^{-2 / 3}(N / S)^{-0.1 / D_{f}}$ for aggregates consisting of smaller polystyrene beads $(\mathrm{R}=0.19 \mu \mathrm{m})$. Soos et al. [11] reinterpreted experimental results by Selomulya et al. for $\dot{\gamma}=64 \mathrm{~s}^{-1}$ and $\mathrm{R}=0.405 \mu \mathrm{m}$ : they obtained $\tau=520 \mathrm{~s}$. So, Baldyga [15] and Selomulya [14] investigations showed very different relaxation time values for $\dot{\gamma}=100 s^{-1}$. To compare $t_{F}$ calculated from Eq.38 with the experimental value $\tau, \Phi_{0}$ in Eq.38 is estimated for fractal aggregates with $\mathrm{D}_{\mathrm{f}}=1.8$. Applying Eq.38 to the data of Selomulya [14] leads to a $t_{F}$ value in the range [60s, 120s] whereas Selomulya found out 30s. Applying Eq.38 to the data handled by Soos [11] leads to a $t_{F}$ value in the range [140s, 250s] whereas Soos found out 520s. The orders of magnitude are similar, but the consistence of the various experimental data and of the calculated values (Eq.38) is not yet definitive.

Restructuring competes with the aggregation itself. In an aggregation process the morphology of aggregates is the result of the complicated coupling between several phenomena: aggregates collide to form a larger aggregate that partially restructures until the next collision. The suspension dynamics can be studied if the aggregates are described by at least two internal variables (for instance, particle size and porosity). A bivariate population balance equation (PBE) may manage the population density with two internal variables. Since the change of the particle size appears under the form of a growth law in the PBE, the restructuring rate (Eq.37b) may appear with the same status [28]. Such a modelling will be applied in the near future.

\section{Acknowledgments}

We acknowledge the Agence Nationale de la Recherche for support of this research. 


\section{References}

[1] M. Elimelech, J. Gregory, X. Jia, R. Williams, Particle deposition and aggregation, measurement, modelling and simulation, Butterworth-Heinemann Ltd, Oxford, 1995.

[2] H.S. Mumtaz, M.J. Hounslow, N.A. Seaton, W.R. Patterson, Orthokinetic aggregation during precipitation, Trans IChemE, 75A, p152, 1997

[3] Y. Xiong and S.E. Pratsinis, Formation of agglomerate particles by coagulation and sintering: Part1 : a two-dimensional solution of the population balance equation, J. Aerosol Sci., 24, p283, 1993

[4] G.D. Ulrich and N.S. Subramanian, Coalescence as a rate-controlling process, Combustion Science \& Technology, 17, p119, 1977

[5] T. Johannessen, S.E. Pratsinis \& H. Livbjerg, Computational fluid-particle dynamics for the flame synthesis of alumina particles, Chem. Eng. Sci, 55, p177, 2000

[6] G. Yang and P. Biswas, Computer simulation of the aggregation and sintering restructuring of fractal-like clusters containing limited numbers of primary particles, J. Colloid \& Interf. Sci., 211, p142, 1999

[7] B. Al Zaitone, H.J. Schmid, W. Peukert, Simulation of structure and mobility of aggregates formed by simultaneous coagulation, sintering and surface growth, Aerosol Sci., 40, p950, 2009

[8] S. di Stasio, "Observation of restructuring of nanoparticle soot aggregates in a diffusion flame by static light scattering”, J. Aerosol Sci., 32(2001)509

[9] Y. Adachi, K. Aoki, "Restructuring of small flocs of polystyrene latex with polyelectrolyte", Colloids and Surfaces A: Physico-chemical and Engineering Aspects, 342(2009) 24

[10] J. Yu, D. Wang, X. Ge, M. Yan, M. Yang, "Flocculation of kaolin particles by two typical polyelectrolytes: A comparative study on the kinetics and floc structures", Colloids and Surfaces A : Physico-chemical and Engineering Aspects, 290(2006)288

[11] M. Soos, J. Sefcik, M. Morbidelli, "Investigation of aggregation, breakage and restructuring kinetics of colloidal dispersions in turbulent flows by population balance modelling and static light scattering", Chem. Eng. Sci., 61(2006)2349

[12] M. Kostoglou, A.G. Konstandopoulos, S.K. Friedlander, "Bivariate population dynamics simulation of fractal aerosol aggregate coagulation and restructuring”, Aerosol Sci. 37(2006)1102 
[13] C. Selomulya, R. Amal, G. Bushell, T.D. Waite, "Evidence of shear rate dependence on restructuring and breakup of latex aggregates", J. Coll. Interf. Sci., 236(2001)67

[14] C. Selomulya, G. Bushell, R. Amal, T.D. Waite, "Understanding the role of restructuring in flocculation: The application of a population balance model", Chem. Eng. Sci., 58(2003)327

[15] J. Baldyga, W. Orciuch, L. Makowski, M. Malski-Brodzicki, K. Malik, "Break up of nano-particle clusters in high-shear devices”, Chem. Eng. Progress, 46(2007)851

[16] A. Dalis, S.K. Friedlander," A lattice chain model for the thermal restructuring of nanoparticle chain aggregates", Aerosol Sci. 36(2005)27

[17] L. Gmachowski, "Estimation of the dynamic size of fractal aggregates", Colloids and surfaces, A: Physicochemical and Engineering Aspects 170(2000)209

[18] L. Gmachowski, "Aggregate restructuring and its effect on the aggregate size distribution", Colloids and surfaces, A: Physicochemical and Engineering Aspects 207(2002)271

[19] M. Kostoglou, A.G. Konstandopoulos, "Evolution of aggregate size and fractal dimension during Brownian coagulation”, Aerosol Sci. 32(2001)1399

[20] K. Higashitani, K. Iimura, H. Sanda, "Simulation of deformation and breakup of large aggregates in flows of viscous fluids", Chem. Eng. Sci., 56(2001)2927-2938

[21] M. Zeidan, B.H. Xu, X. Jia and R.A. Williams, "Simulation of aggregate deformation and breakup in simple shear flows using a combined continuum and discrete model", Trans IChemE, Part A, 85(2007)1645-1654

[22] Y. Tatek, E. Pefferkorn, "Cluster-cluster aggregation controlled by the number of intercluster connections: kinetics of aggregation and cluster mass frequency", J. Colloid \& Interf. Sci., 2004, 278, p361

[23] G.K. Batchelor, Slender-body theory for particles of arbitrary cross-section in Stokes flow, J. Fluid. Mech., 44, p419, 1970

[24] S.N. Rogak and R.C. Flagan, Stokes drag on self-similar clusters of spheres, J. Colloid \& Interf. Sci., 134, p206, 1990

[25] G.R. Zeichner and W.R. Schowalter, Use of trajectory analysis to study stability of colloidal dispersions in flow fields, AIChE J., 23, p243, 1977

[26] G. Narsimhan, Model for drop coalescence in a locally isotropic turbulent flow field, J. of Colloid \& Interface Science, 272(2004)197-209 
[27] V. Oles, Shear-induced aggregation and break-up of polystyrene latex particles, J. of Colloid \& Interface Science, 154(1992)351-358

[28] F. Gruy, "Population balance for aggregation coupled with morphology changes",

Colloids and surfaces, A: Physicochemical and Engineering Aspects 374(2011)69-76 


\begin{tabular}{|l|l|l|l|l|l|}
\hline $\mathrm{K}$ & 2 & 4 & 6 & 10 & 20 \\
\hline $\mathrm{P}$ & 0.17 & 0.33 & 0.38 & 0.44 & 0.54 \\
\hline $\mathrm{T}$ & 13.2 & 33.5 & 49.7 & 66.6 & 134 \\
\hline
\end{tabular}

Table 1: effect of the maximum size of the aggregate on the restructuring kinetics 


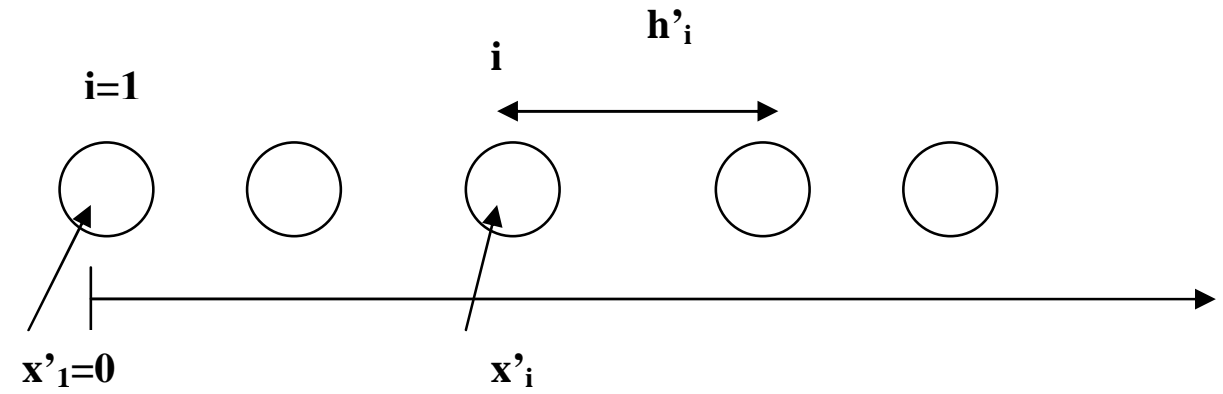

Figure 1: chain of $N$ mono-sized primary particles $(t=0)$ 


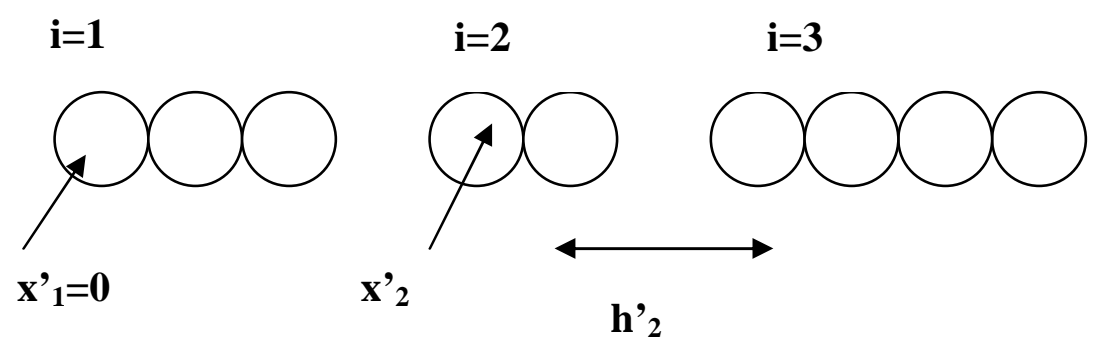

Figure 2: chain of $N_{\mathrm{a}}$ clusters $(t \neq 0)$ 


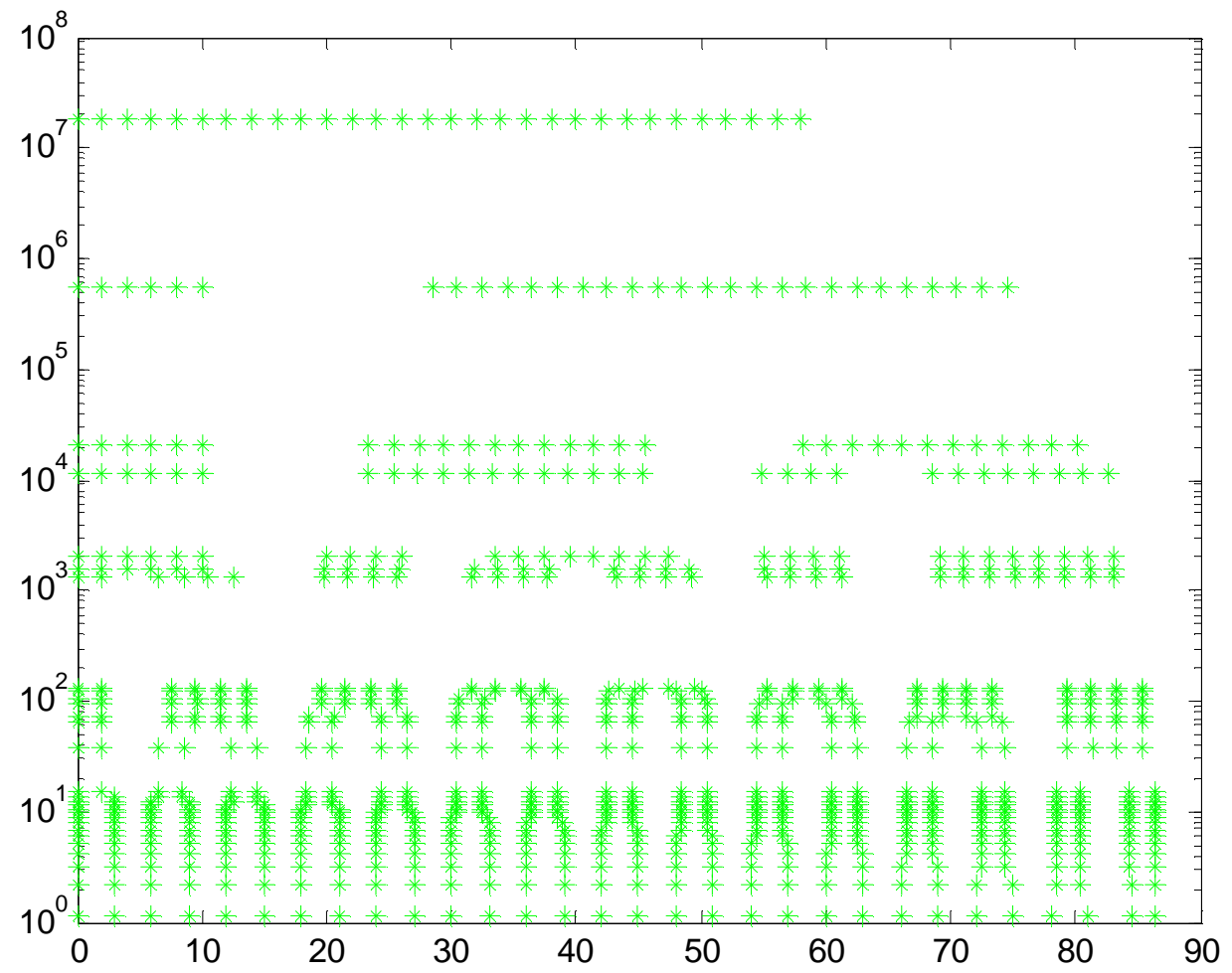

Figure 3: change with time of the chain morphology $(N=30)$. The vertical axis represents the dimensionless time. 


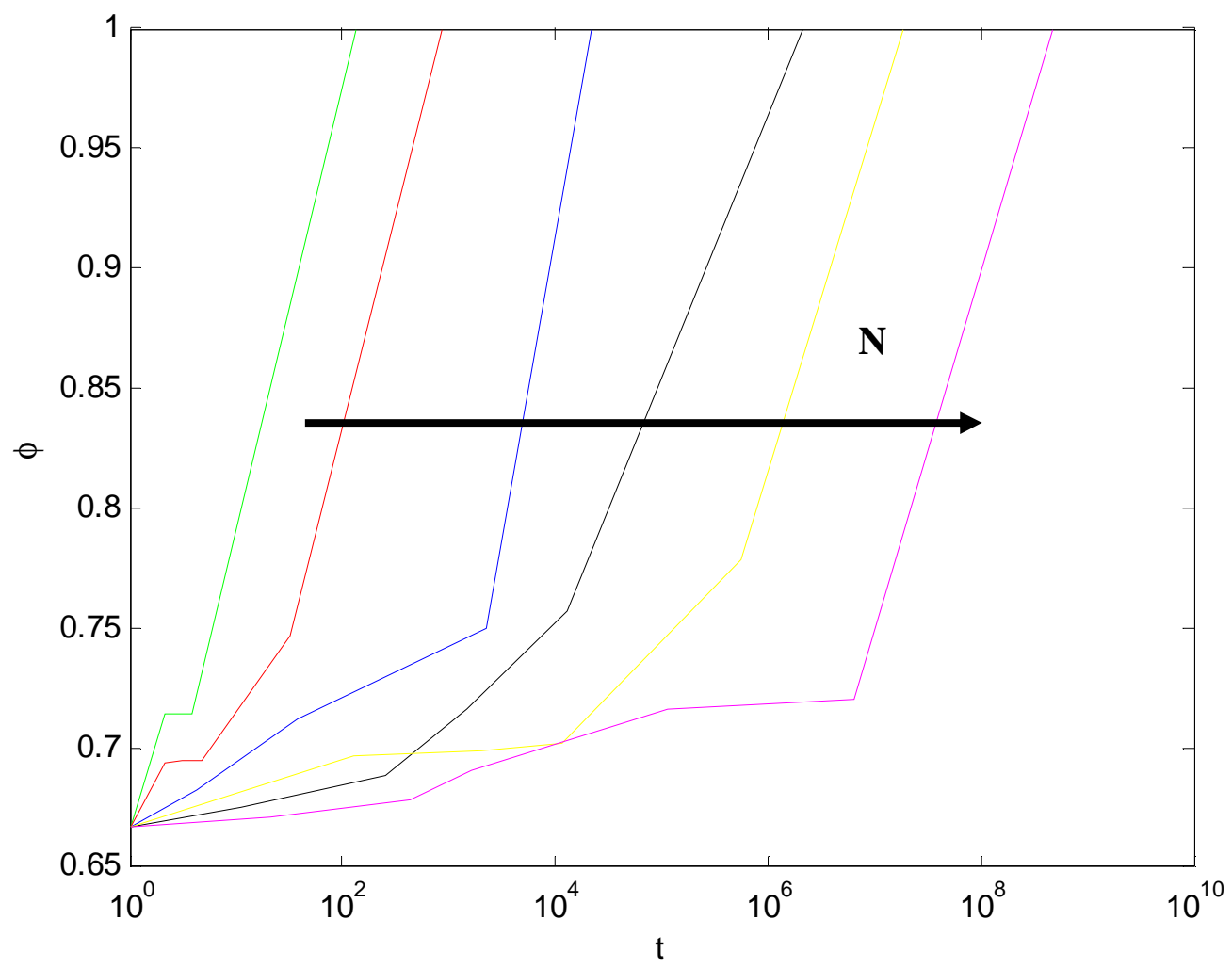

Figure 4: solid volume fraction as a function of dimensionless time for various $N$ values 


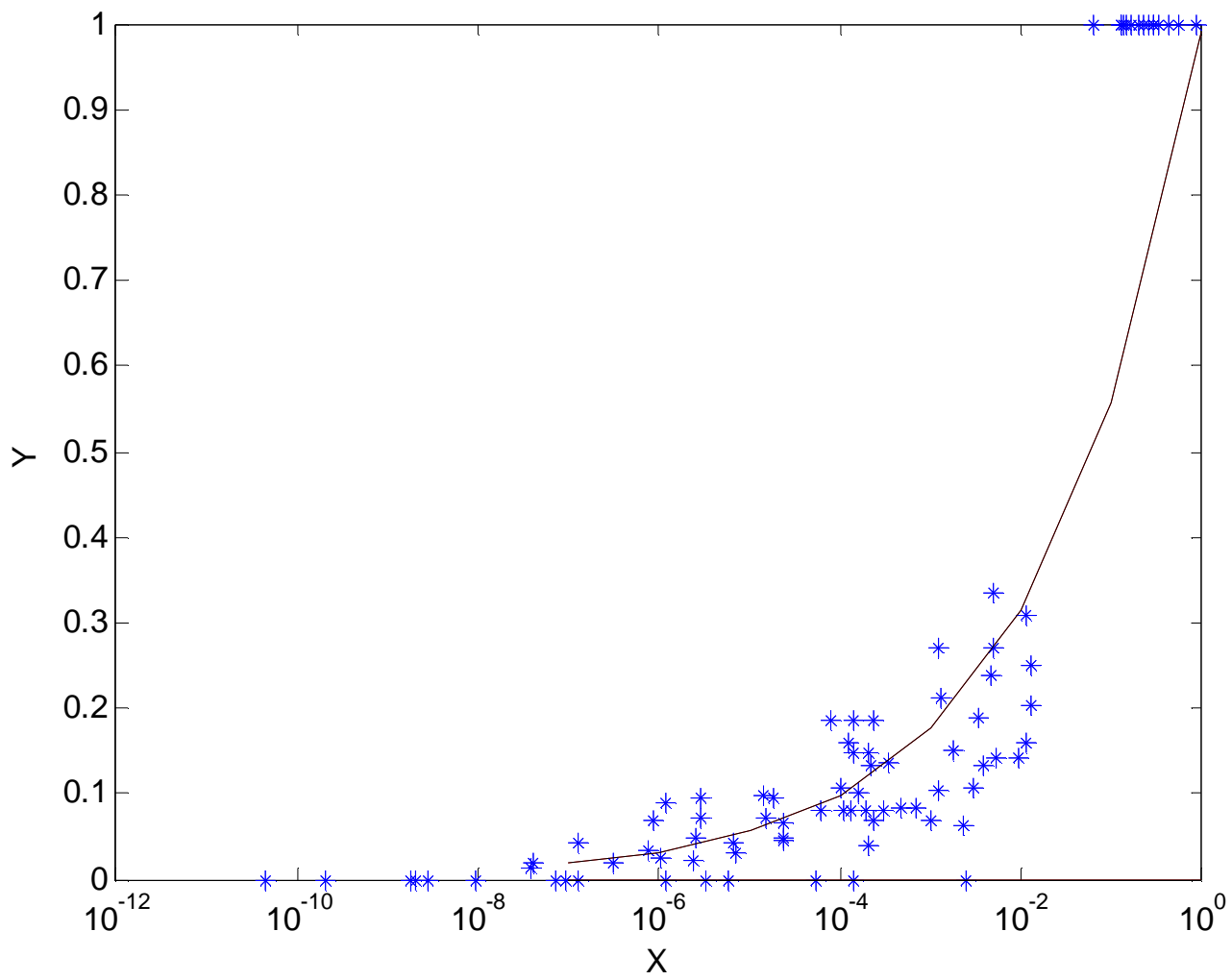

Figure 5: normalized solid volume fraction versus dimensionless time $X$. The dots correspond to simulations. 


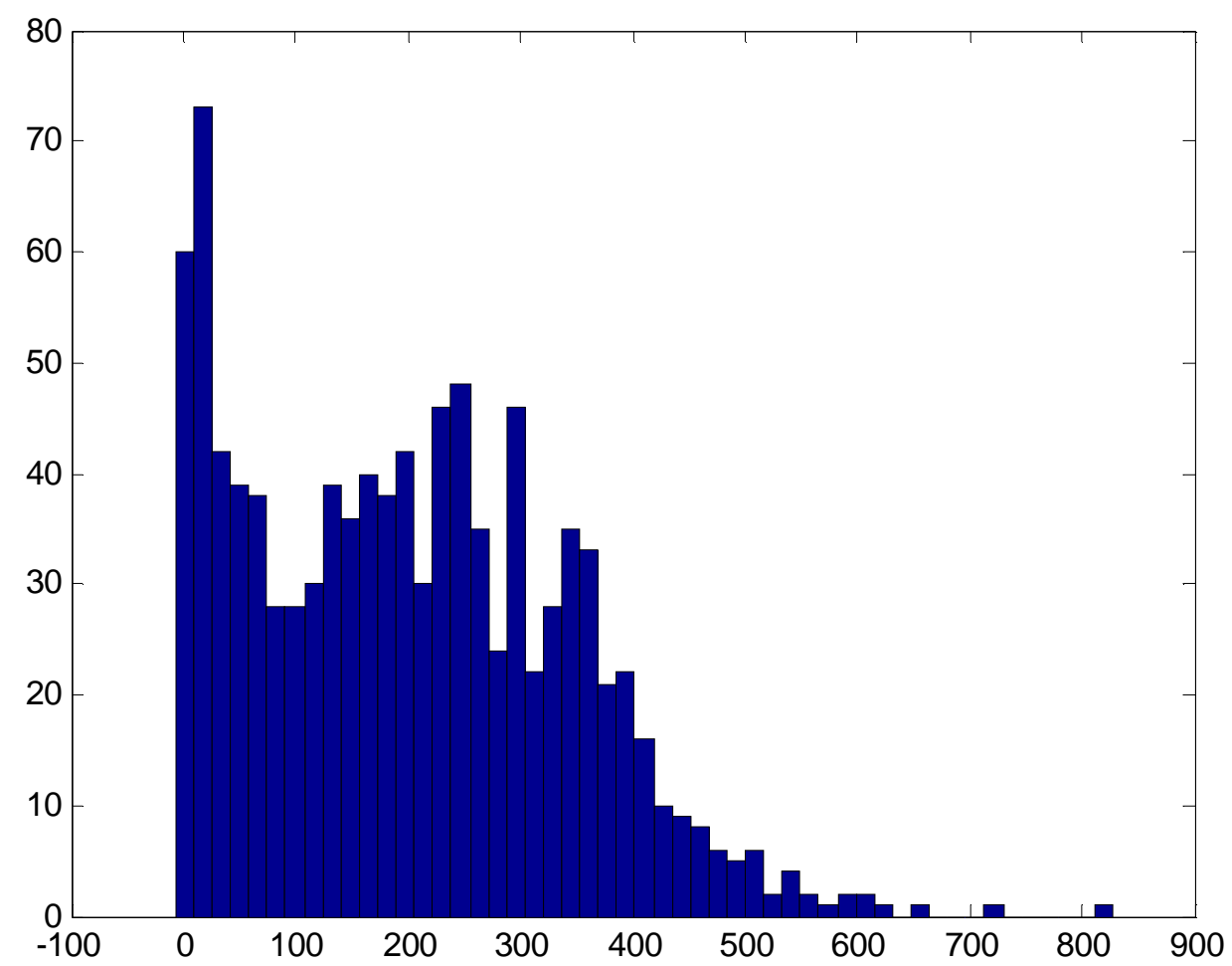

Figure 6a: distribution density of the total shrinking time $(N=4)$ 


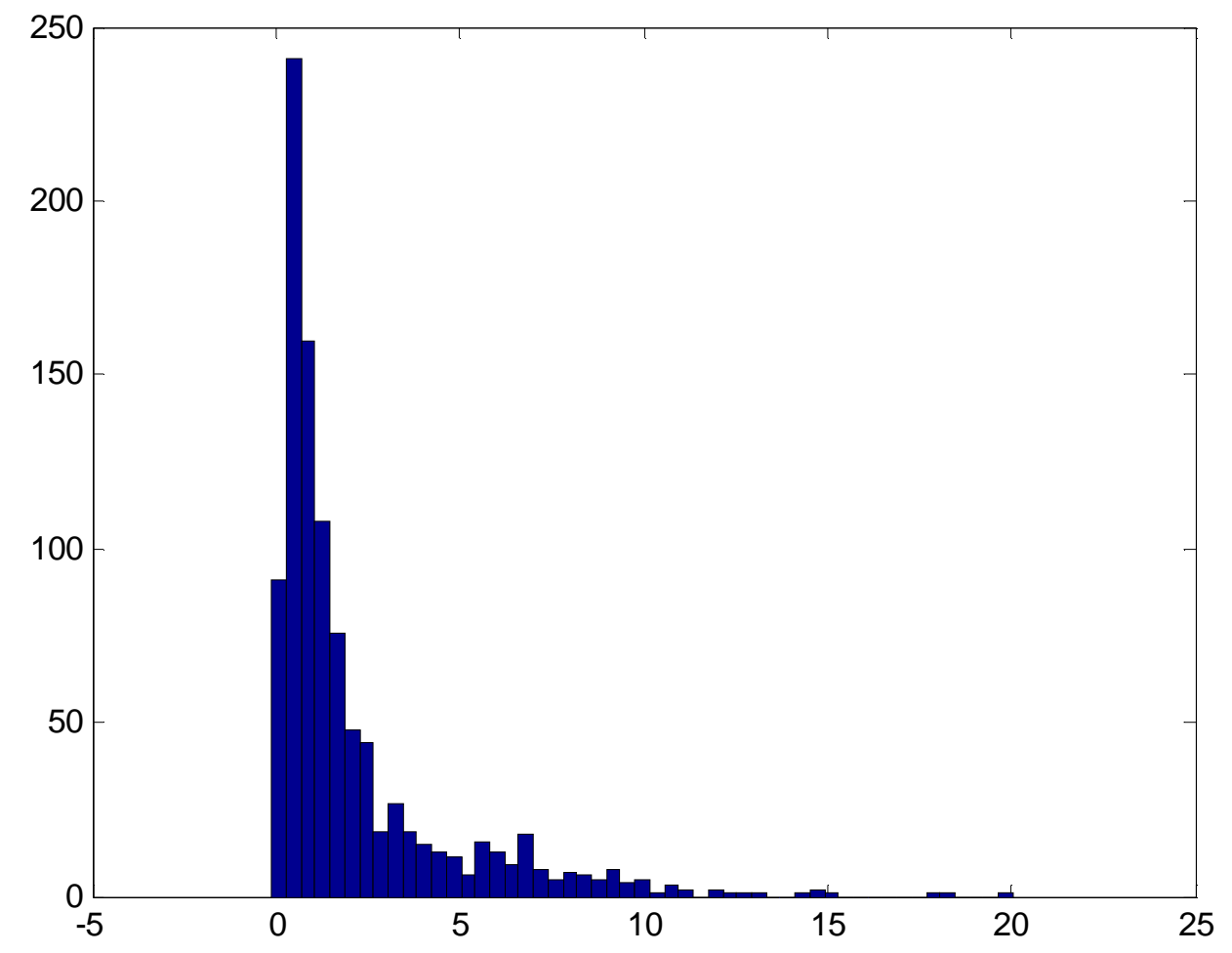

Figure 6b: distribution density of the shrinking time at an intermediate event when the aggregate consists of two clusters $(N=4)$ 


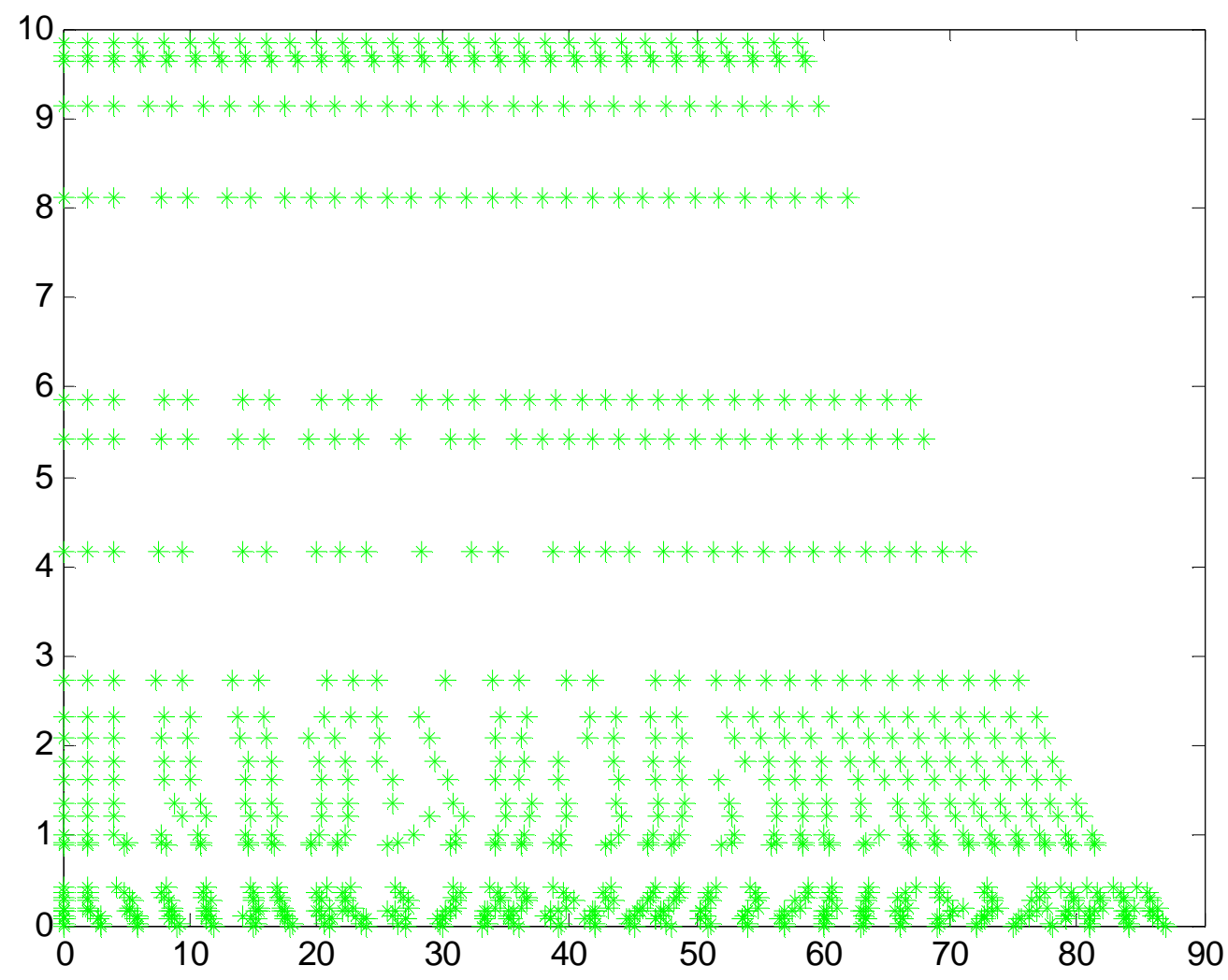

Figure 7: change with time of the chain morphology $(N=30)$. The vertical axis represents the dimensionless time. 


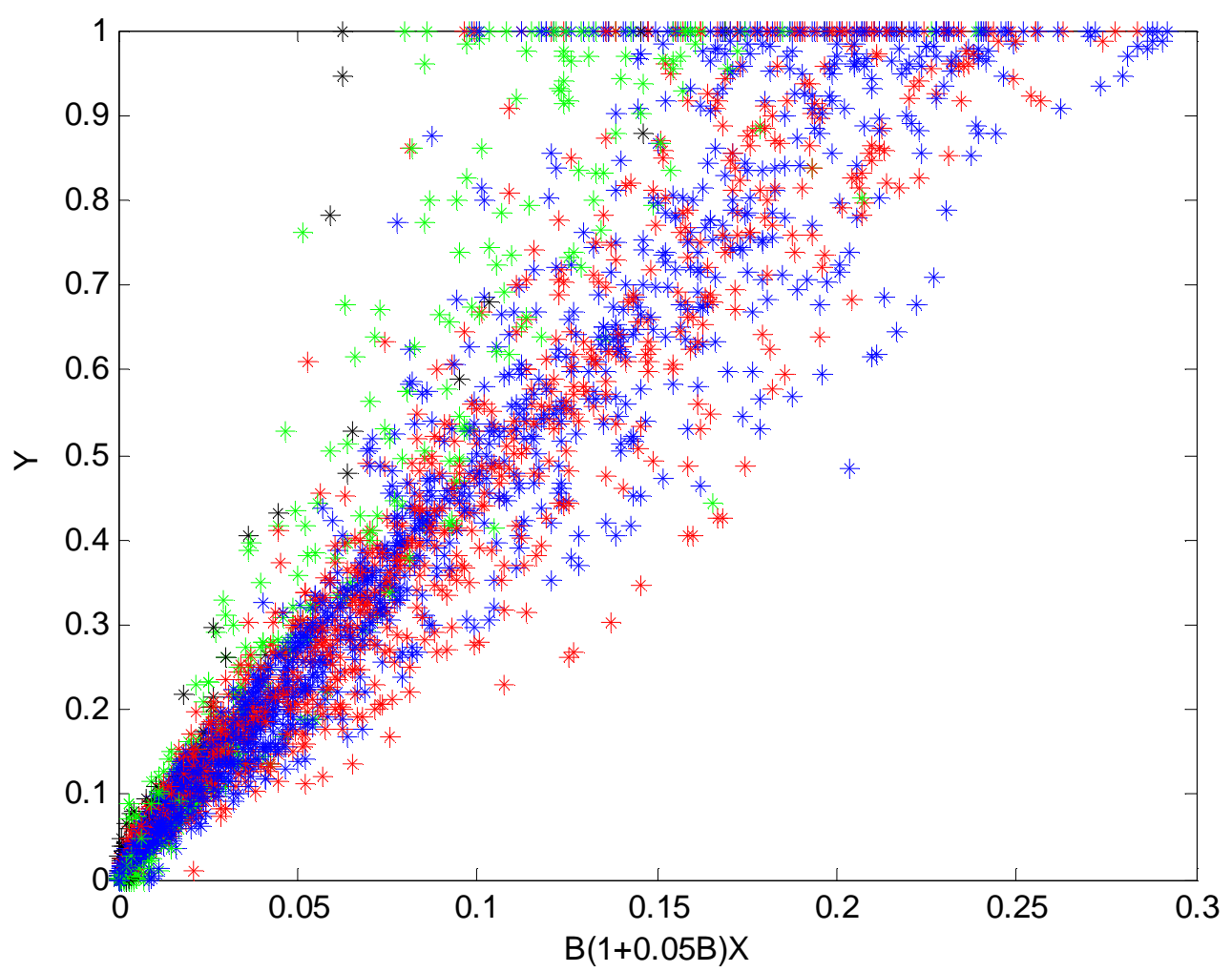

Figure 8a: restructuring law for an aggregate in a weakly turbulent flow (equidistant interparticle distances); $B<25$. fluctuating shear at the surface of the aggregate
$\mathrm{B}<0.1$ : black
$0.1<\mathrm{B}<1$ : green
$1<\mathrm{B}<10$ : blue
B>10: red 


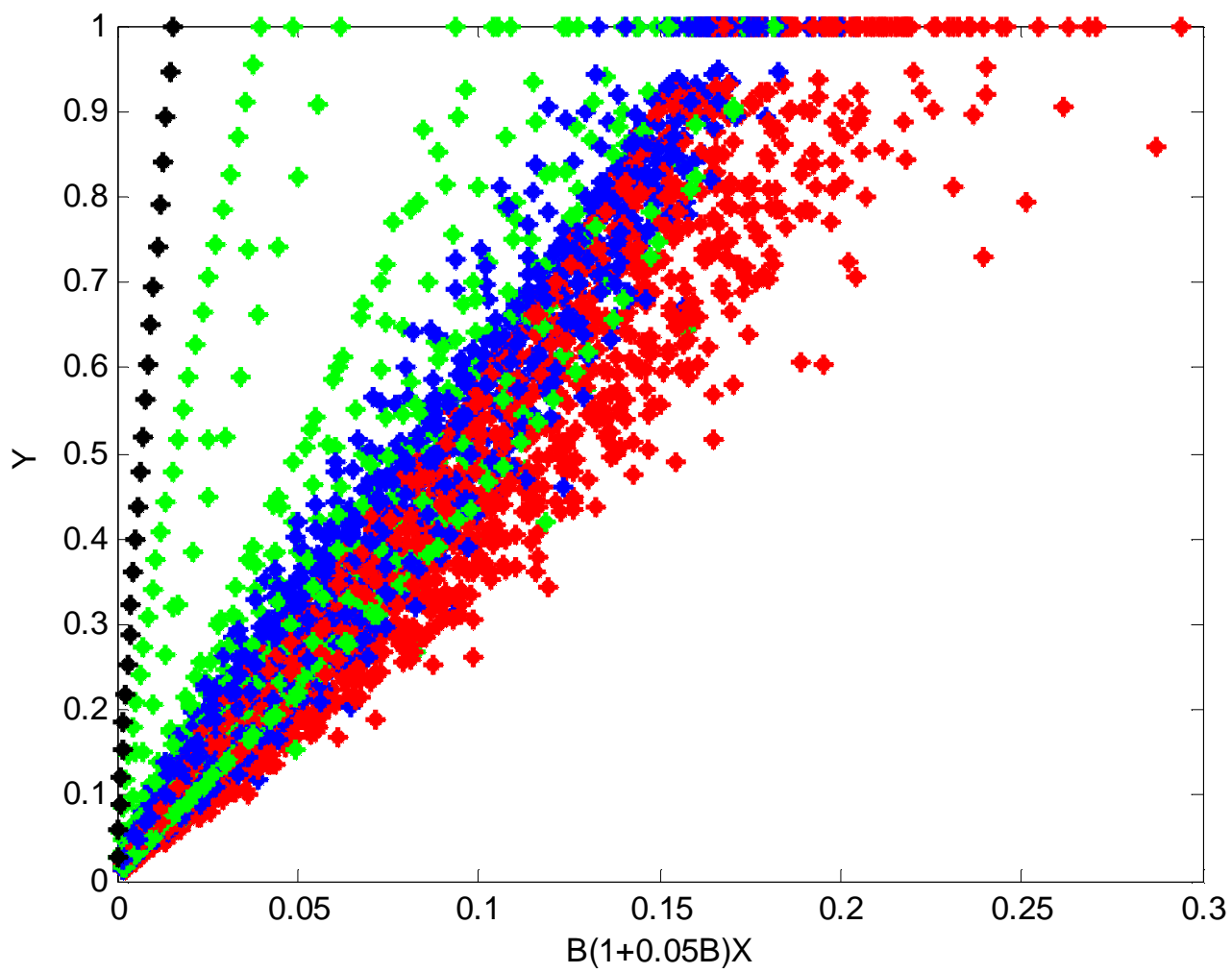

Figure 8b: restructuring law for an aggregate in a weakly turbulent flow (equidistant interparticle distances) following Eqs. 34-35; $B<25$.

$\mathrm{B}<0.1$ : black $\quad 0.1<\mathrm{B}<1$ : green $\quad 1<\mathrm{B}<10$ : blue $\quad \mathrm{B}>10$ : red 